Research Paper

\title{
Hypoxia-Mimicking Cobalt-Doped Borosilicate Bioactive Glass Scaffolds with Enhanced Angiogenic and Osteogenic Capacity for Bone Regeneration
}

\author{
Zhengwei Deng ${ }^{1,2^{*}}$, Bocai Lin $^{3 *}$, Zenghui Jiang ${ }^{*}$, Wenhai Huang 5 , Jiusheng Li $^{3}$, Xiangqiong Zeng 3 , Hui \\ Wang $^{3 凶}$, Deping Wang ${ }^{\circledR 凶}$, Yadong Zhang ${ }^{1,6 凶}$ \\ 1. Department of Orthopedics, South Campus of Shanghai Sixth People's Hospital Affiliated of Shanghai University of Medicine\&Health Sciences, 279 \\ zhouzhu road, Shanghai 220120, People's Republic of China. \\ 2. Graduate School, Shanghai University of Traditional Chinese Medicine, Shanghai 201203, People's Republic of China. \\ 3. Laboratory for Advance Lubricating Materials, Shanghai Advanced Research Institute, Chinese Academy of Sciences, Shanghai 201210, People's Republic of \\ China. \\ 4. Department of Orthopedic Surgery, Zhejiang Hospital, Hangzhou 310013, People's Republic of China. \\ 5. School of Materials Science and Engineering, Tongji University, Shanghai 200092, People's Republic of China. \\ 6. Shanghai Fengxian District Central Hospital Affiliated of Southern Medical University, Shanghai 201400, People's Republic of China. \\ *These authors contributed equally to this work.
}

$\triangle$ Corresponding authors: Dr. Yadong Zhang: Tel: +86 21 64369181; Email:zhangyadong6@126.com or Dr. Hui Wang: Tel: +86 21 20350946; Email: hui-wang2014@outlook.com or Prof. Deping Wang: Tel: +86 21 69582134; Email: wdpshk@tongii.edu.cn

(c) Ivyspring International Publisher. This is an open access article distributed under the terms of the Creative Commons Attribution (CC BY-NC) license (https://creativecommons.org/licenses/by-nc/4.0/). See http://ivyspring.com/terms for full terms and conditions.

Received: 2018.12.17; Accepted: 2019.02.23; Published: 2019.04 .22

\begin{abstract}
The osteogenic capacity of synthetic bone substitutes is will be highly stimulated by a well-established functional vascularized network. Cobalt (Co) ions are known that can generate a hypoxia-like response and stimulates the production of kinds of angiogenic factors. Herein, we investigated the mechanism of cobalt-doped bioactive borosilicate $\left(36 \mathrm{~B}_{2} \mathrm{O}_{3}, 22 \mathrm{CaO}, 18 \mathrm{SiO}_{2}, 8 \mathrm{MgO}\right.$, $8 \mathrm{~K}_{2} \mathrm{O}, 6 \mathrm{Na}_{2} \mathrm{O}, 2 \mathrm{P}_{2} \mathrm{O}_{5}$; mol\%) glass scaffolds for bone tissues repairing and blood vessel formation in the critical-sized cranial defect site of rats and their effects on the hBMSCs in vitro were researched. The scaffolds can control release $\mathrm{Co}^{2+}$ ions and convert into hydroxyapatite soaking in simulative body fluids (SBF). The fabircated scaffolds without cytotoxic strongly improves HIF-la generation, VEGF protein secretion, ALP activity and upregulates the expression of osteoblast and angiogenic relative genes in hBMSCs. Eight weeks after implantation, the bioactive glass scaffolds with $3 \mathrm{wt} \%$ $\mathrm{CoO}$ remarkablely enhance bone regeneration and blood vascularized network at the defective site. In conclusion, as a graft material for bone defects, low-oxygen simulated cobalt-doped bioactive glass scaffold is promising.
\end{abstract}

Key words: bioactive glass scaffolds, cobalt ions, hypoxia-like response, bone regeneration, blood vascularized network

\section{Introduction}

Nowadays, repairing critical-sized defects caused by tumor resection and congenital skeletal abnormalities is still a challenge in orthopedic surgery. Although the allogenetic and autologous bone implants are widely used, they suffer many limitations such as donor site morbidity, risk of disease transmission and high cost. ${ }^{[1]}$

So, the synthetic implants drew many attentions recently, for providing the requisite threedimensional (3D) architecture to serve as a scaffold, a microenvironment for cell phenotype maintenance, and the indispensable osteogenic capacity for bone regeneration. ${ }^{[2][3][4]}$ However, only limited success has been achieved so far in healing large bone defects using synthetic biomaterials. In general, vascularization and new bone ingrowth are often limited to the 
periphery of the implants. ${ }^{[5][6]}$ In order to improve in vivo the performance of synthetic biomaterials, the development of 3D scaffolds with enhanced angiogenic and osteogenic capacity is receiving considerable attention. ${ }^{7]}$

It is well-known that the osteogenic capacity of implanted scaffolds is signally dependent on the formation of vascularized network because newly formed blood vessels provide oxygen and nutrients, facilitate metabolic waste removal and deliver renewable progenitor cells. ${ }^{[8][9]}$ previously, it has been shown that a low oxygen (hypoxic) microenvironment can promote tissue development and repair via progenitor cell recruitment, differentiation and blood vessel formation. ${ }^{[10][11][12] ~ H I F-1 \alpha}$ is a protein that stimulates the expression of a variety of angiogenic factors such as vascular endothelial growth factor (VEGF), stromal-derived factor 1 (SDF-1), basic fibroblast growth factor (bFGF) and transforming growth factor beta (TGF- $\beta$ ).[13] [14][15][16] Hypoxia can stabilize HIF-1a expression by the application of prolyl hydroxylase enzyme (PHD) inhibitors such as desferrioxamine (DFO), L-mimosine (L-mim), dimethyloxalylglycine (DMOG), and $\mathrm{Co}^{2+}$ ions. [17][18][19][20]

As the only PHD inhibitor in ionic form, $\mathrm{Co}^{2+}$ ions have been shown to activate the HIF-1a pathway to promote a hypoxia-like response and to affect the angiogenesis with osteogenesis, which would be of great interest for applications in bone tissue engineering.[10][22] Compared to genetic engineering or recombinant proteins methods for improving angiogenesis, the use of $\mathrm{Co}^{2+}$ ions has several advantages such as low cost, long-term stability with low regulatory burden and potentially greater safety. Cobalt can be incorporated into bioactive glass in the manufacture process, when the fabricated glass degrades it can control release then doped ions at a therapeutically appropriate rate. Consequently, the very capacity to stimulate angiogenesis can be combined with the well-known osteogenic capacity of bioactive glasses.

Azevedo, et al. have found that the released trend of $\mathrm{Co}^{2+}$ ions was dependent on its Co content, indicating the potential for controlled delivery of $\mathrm{Co}$ ions within therapeutically active doses.[6] Hoppe et al. fabricated Co-containing 1393 bioactive glassbased scaffolds and revealed that the compressive strength of scaffolds $>2 \mathrm{MPa}$ as well as excellent in vitro bioactivity.[21] $\mathrm{Wu}$ et al. showed that incorporating Co into bioactive glass could activate the proliferation, VEGF secretion, and upregulate the expression of bone-related gene of BMSCs. However, they did not investigate whether the Co-containing scaffolds could induce a hypoxia function in large bone defects in vivo.[22]

In previous studies, we informed that borosilicate bioactive scaffolds possessed containable percent conversion to hydroxyapatite (HA) and offered an amazing transmission system for inorganic ions in treating bone defects. ${ }^{[23][24]}$ In this study, we manufacture porous 3D scaffolds by doping Co (0.5-3.0 wt \% CoO) into rooted borosilicate bioactive scaffolds and we have evaluated the effects of the Co on vascular formation, bone repairing and the responses of hBMSCs in rat calvarial defects in vitro and vivo. The responses of hBMSCs cultured on the scaffolds, include cell adhesion, proliferation, VEGF secretion, ALP activity, HIF-1a expression, and the expression levels of osteogenesis and angiogenesis relative genes. Additionally, micro-ct and immunohistochemical and histological methods are applied to analyze the angiogenesis and bone regeneration of the calvarial defects in rats 8 weeks after implantation.

\section{Experimental section}

\section{Synthesis and characterization of the as-prepared Co-BG scaffolds}

The primary borosilicate glass (designated $\mathrm{BG}$ ) was comprised of a borosilicate composition $\left(36 \mathrm{~B}_{2} \mathrm{O}_{3}\right.$, $22 \mathrm{CaO}, 18 \mathrm{SiO}_{2}, 8 \mathrm{MgO}, 8 \mathrm{~K}_{2} \mathrm{O}, 6 \mathrm{Na}_{2} \mathrm{O}, 2 \mathrm{P}_{2} \mathrm{O}_{5} ; \mathrm{mol} \%$ ) and the glass scaffolds composed of $\mathrm{BG}$ and $\mathrm{BG}$ mingled with $0.5,1.0$ and $3.0 \mathrm{wt} \% \mathrm{CoO}$ (designated 0.5Co-BG; 1Co-BG, and 3Co-BG, respectively) were generated via using traditional melting and casting methods. Additionally, the scaffolds applied in this study were created by a foam replication technique.[25] The morphology of the above obtained scaffolds was characterized by field-emission scanning electron microscopy (FESEM) with a SU8020 (Hitachi, Japan). XRD (X-ray diffraction, Rigaku, Tokyo, Japan) were used to represent a glass powder and the existence of any crystal phases. Mechanical testing machine (CMY6104 SANS) was applied to testify the compressing force of cylindrical scaffolds, at a cross-head speed of $0.5 \mathrm{~mm} / \mathrm{min}$ and a $1 \mathrm{kN}$ load cell.

\section{Degradation of as-prepared scaffolds}

The bioactivity performance, degradation process and mineralization of these foregoing as-prepared scaffolds were determined by soaking in SBF (simulated body fluid). A ratio of $0.1 \mathrm{~g}$ to $10 \mathrm{ml}$ for as-fabricated scaffolds immersion in SBF solution was applied to estimate the sample degradation process by monitor the relative weight loss changing of immersion between $\mathrm{BG}$ and Co-BG sample and $\mathrm{pH}$ value changing of SBF immersion. The contrastive concentration of dissolved ions controlled-release from BG and Co-BG scaffolds in SBF, such as silicate 
and $\mathrm{Ca}$ ions, was tested by ICP-AES (inductivelycoupled plasma atomic emission spectroscopy). At long last, the superficial HA formationon of these scaffolds was characterized through FESEM and XRD.

\section{Cellular evaluations of Co-BG scaffolds in vitro}

We isolated and cultured human bone marrow mesenchymal stem cells (hBMSCs) using previously described experimental methods. ${ }^{[26]}$ The bone marrow suspension was then removed with a $70 \mathrm{~mm}$ cell strainer, the initial cell viability was determined by trypan blue exclusion and the number of cells was measured by a hematometer. Cell culture in Durbeck's modified Eagle's medium (DMEM) (GIBCO; Invitrogen Pty Ltd. Australia) supplemented with $10 \%$ fetal calf serum (FCS)(In Vitro Technologies, Australia) with $5 \% \mathrm{CO}_{2}$ at $37^{\circ} \mathrm{C}$ in a humidified atmosphere. The medium was changed after 48 hours and thereafter every 3 days. Based on previous research 4-7 generations of cells were used for subsequent cellular experiments. [27]

The scaffolds with seeded hBMSCs incubated in DMEM supplemented with $10 \%$ FBS, $100 \mu \mathrm{g} / \mathrm{mL}$ of streptomycin, and $100 \mathrm{U} / \mathrm{mL}$ of penicillin at $37^{\circ} \mathrm{C}$ in a comfortable environment of $5 \% \quad \mathrm{CO}_{2}$. For the morphology, the scaffolds with hBMSCs attached was covered with gold layer and observed by FESEM at the acceleration voltage of $15 \mathrm{kV}$. The proliferation of the cultured hBMSCs was evaluated by CCK-8, and for testing the absorbance value at $450 \mathrm{~nm}$. The results were about the optical density of the aliquots minus the absorbance of the blank wells. Meanwhile, the differentiation of the hBMSCs was determined by testing the ALP activity that was measured on days 7 and 14 after seeding $1 \times 10^{5} \mathrm{hBMSC}$ on each scaffold of a group $(n=3)$, and the deserved results were tested via the absorbance at $405 \mathrm{~nm}$.

The relative expression of representative osteogenesis and angiogenic related genes, including VEGF, SDF-1, bFGF, and bone morphgenetic protein-2 (BMP-2), in hBMSCs were also investigated using qRT-PCR. The cells were seeded at a density of $2 \times 10^{4}$ cells/well, cultured for 7 or 14 days and harvested using TRIzol Reagent to extract the RNA. The achieved RNAs were reversely transcribed into complementary DNA (cDNA) using RevertAid First Strand cDNA Synthesis Kit and the qRT-PCR analysis was performed on an ABI Prism 7300 Thermal Cycler using SYBR Green detection reagent. Against the housekeeping gene GAPDH, the relative expression of the genes of interest was normalized. The VEGF secretion of the hBMSCs were tested by enzyme-linked immunosorbent (ELISA) assays, and the HIF-1a protein was analyzed by the western blot test.

\section{The capacity of osteogenesis and angiogenic of Co-BG scaffold in vivo}

The animal treatment procedures were carried out according to the guidelines approved by the Shanghai Jiao Tong University Ethics Committee. Thirty-six male Sprague Dawley rats (Three months old; 250-300 g) were used to fabricate rat calvarial defects models. Thereinto, unfilled defects were used as control groups, 3 Co-BG scaffolds and undoped BG scaffolds with $1.5-2 \mathrm{~mm}$ in height and $5 \mathrm{~mm}$ in diameter, the positive, negative groups, respectively. After 8 weeks of post operation, the rats were sacrificed after surgery.

A Micro-CT scanner (Skyscan 1176, Kontich, Belgium) was used to reconstruct images using software, and by which, the relative bone volume fraction (BV/TV) and the bone mineral density (BMD) were affirmed. The Van Gieson's picrofuchsin staining was applied to analyze the formative evaluation of new bone tissue. Histological staining observation image was examined by Image Pro Plus $^{\mathrm{TM}}$ (Media Cybernetics, Silver Springs, MD). To obtain new bone formation and mineralization rate, we were put to death in 8 weeks of polychrome sequential fluorescent labeling in rats, 2 weeks, 4 weeks and 6 weeks after surgery, after the ether anesthesia to the animals and intraperitoneal injection of fluorescent composed of $30 \mathrm{mg} / \mathrm{kg}$ alizarin red (AL) (Sigma, USA), $25 \mathrm{mg} / \mathrm{kg}$ tetracycline (TE), and $20 \mathrm{mg} / \mathrm{kg}$ calcein (CA) (Sigma, USA).

To evaluate blood vessel formation at 8 weeks post-surgery, the rats were perfused with Microfil (Microfil MV-122; Flow Tech, Carver, MA) after euthanasia. When they were decalcified in 10\% ethylenediaminetetraacetic acid (EDTA; Sinopharm Chemical Reagent Co. Ltd) for 2 weeks, the calvarial samples were embedded in paraffin and sectioned at 5 $\mu \mathrm{m}$ at the central area of the defect. Immunohistochemistry and immunofluorescence staining for CD31 (ab24590, 1:200; Abcam) and osteocalcin (OCN, ab13420, 1:100; Abcam) were then performed in the sections from each group.

\section{Statistical analysis}

All data were expressed as means \pm standard deviation (SD) and were analyzed using one-way ANOVA for independent sample. The criteria for statistical significance were ${ }^{*} p<0.05$.

\section{Results}

\section{Characterization of Co-BG scaffolds}

FESEM analysis demonstrated that the Co-doped BG and simple BG scaffolds had no observable difference in micro-texture (Fig.1 A1, B1, 
C1 and D1). The Co-doped scaffolds showed a stepped-up purple color when the doped amount was increased. The porosity of as-fabricated scaffolds was around $80 \pm 3 \%$ and pores size were about 200-500 $\mu \mathrm{m}$. Regrettably, the mechanical behavior of these scaffolds was decreased with Co-doped amount increase, from $3.3 \pm 0.4 \mathrm{MPa}$ to $2.4 \pm 0.3 \mathrm{MPa}$ for $\mathrm{BG}$ to 3Co-BG scaffolds (Fig. 2C), while these data still luckily keep in the range of reported value for human trabecular bone, ${ }^{[28]}$ which means the scaffolds could also bear the body weight.

\section{Degradation capacity of as-prepared Co-BG scaffolds}

High magnification FESEM images revealed a smooth surface of the scaffolds, while a particulate microstructure change was caught after immersion (Fig. 1). In the meantime, the element analysis of these particle is equivalent to 1.67 of stoichiometric HA from EDS results. The XRD of the synthetic scaffolds showed a broad band, indicating a typical amorphous glass (Fig. 2A) for the as-prepared scaffolds. However, the data of the soaked scaffolds implied a peak corresponding to reference HA, proving the transformation of bioactive glass to HA (Fig. 2B).

During the degradation process, the basic glass scaffold could release some ions and entail some variation on the soaking medium. The weight loss of the scaffolds changed rapidly in the first 14 days and kept a nearly constant value later (Fig. 2D). At 8 weeks, after the immersion process, the final weight loss was $44 \pm 2 \%, 38 \pm 3 \%, 35 \pm 3 \%$ and $32 \pm 3 \%$ for the BG and Co-BG scaffolds. The accumlated amount of $\mathrm{Co}^{2+}$ ions in the SBF also increased rapidly during the first 14 days and reached a nearly almost steady value thereafter, except for the 3Co-BG scaffolds for which the release of $\mathrm{Co}^{2+}$ ions continued until the experiments were stoped at 8 weeks (Fig. 2E). These results exhibited that the cumulative amount of released $\mathrm{Co}^{2+}$ increased as the raising doping amount of Co. The highest amount of Co released, at the first day, is 4.7, 7.0, $21.1 \mathrm{ppm}$ and the final accumulated amount of $\mathrm{Co}^{2+}$ ions released was $44 \%$ (14.4 ppm), 37\% (23.8 $\mathrm{ppm})$ and $36 \%$ (70.7 ppm) of the total doping amount, for the $0.5 \mathrm{Co}-\mathrm{BG}, 1 \mathrm{Co}-\mathrm{BG}$ and $3 \mathrm{Co}-\mathrm{BG}$ scaffolds.
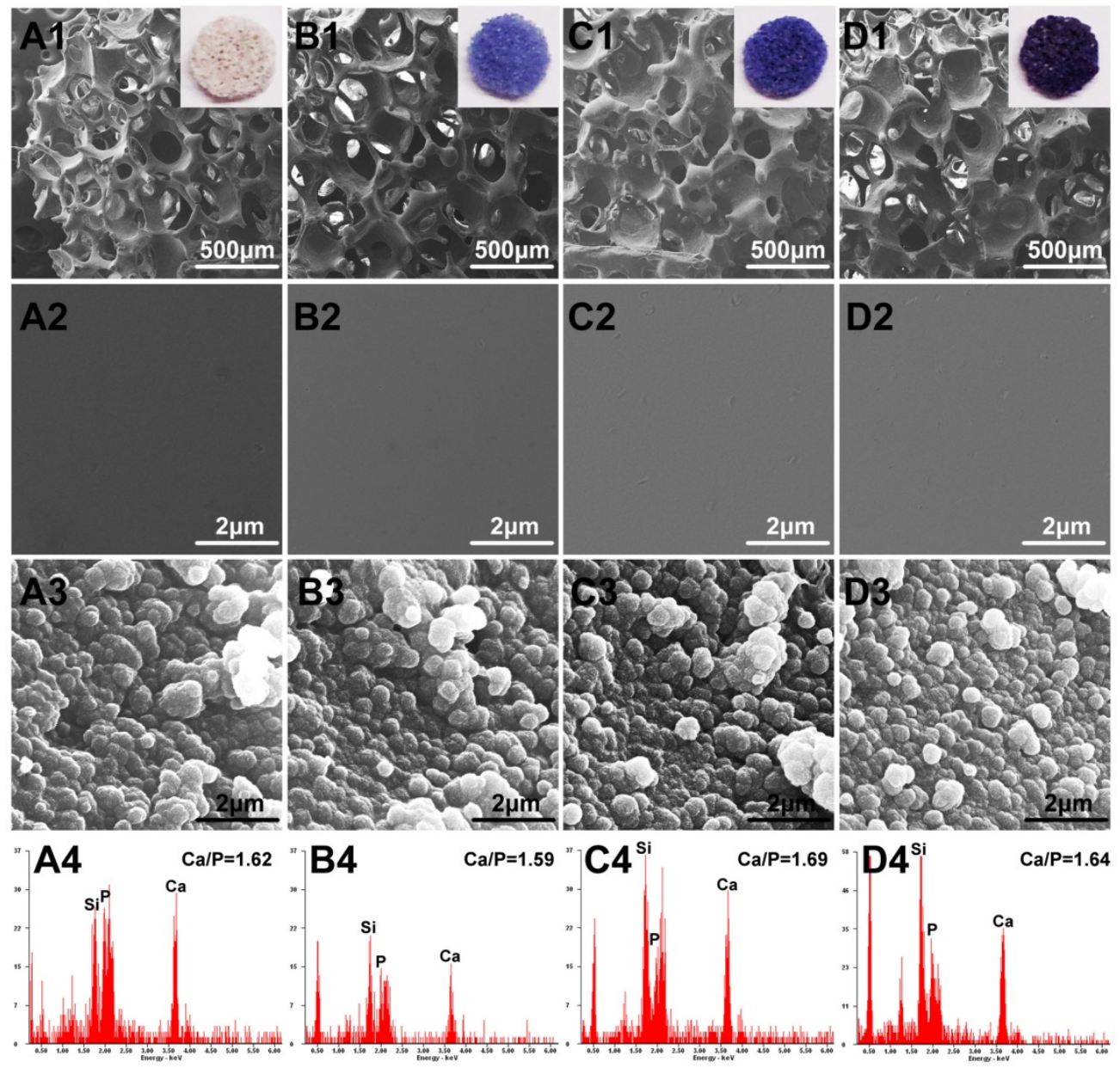

Figure 1. Optical images and FESEM images for the BG (A1, A2), 0.5Co-BG (B1, B2), 1Co-BG (C1, C2) and 3Co-BG (D1, D2) scaffolds before soaking in SBF; SEM images for the BG (A3), 0.5Co-BG (B3), 1Co-BG (C3) and 3Co-BG (D3) scaffolds after soaking in SBF for 90 days; EDS analysis for the BG (A4), 0.5Co-BG (B4), 1 Co-BG (C4) and 3Co-BG (D4) scaffolds after soaking in SBF for 90 days. Magnification: $\times 100$ for A1, B1, C1, D1; $\times 40,000$ for A3, B3, C3 and D3, A4, B4, C4 and D4. 


\section{Cell responses of hBMSCs on Co-BG scaffolds}

From the FESEM images, we can easily find that cultured hBMSCs can spread well on the surfaces of four groups of scaffolds for $72 \mathrm{~h}$ (Fig. 3). Also, the morphology and prominent filopodia of the hBMSCs were clearly observed by these images. Cell proliferation increasing conspicuously of hBMSCs was found in most treatment groups in Fig. 4. The proliferation of hBMSCs on 0.5Co-BG and 1Co-BG scaffolds showed no obvious difference from that of the BG scaffolds, in spite of the cell proliferation on the 3Co-BG scaffolds that was lower than that on BG scaffolds at day 7.

\section{Angiogenesis/osteogenesis related genes expression and VEGF secretion of hBMSCs on Co-BG scaffolds}

As shown in Fig. 4, the 0.5Co-BG, 1Co-BG and 3Co-BG scaffolds significantly enhanced VEGF protein secretion of the hBMSCs. At the same time, HIF-1a expression of the hBMSCs incubated on the Co-doped glass scaffolds also increased with increased Co content.
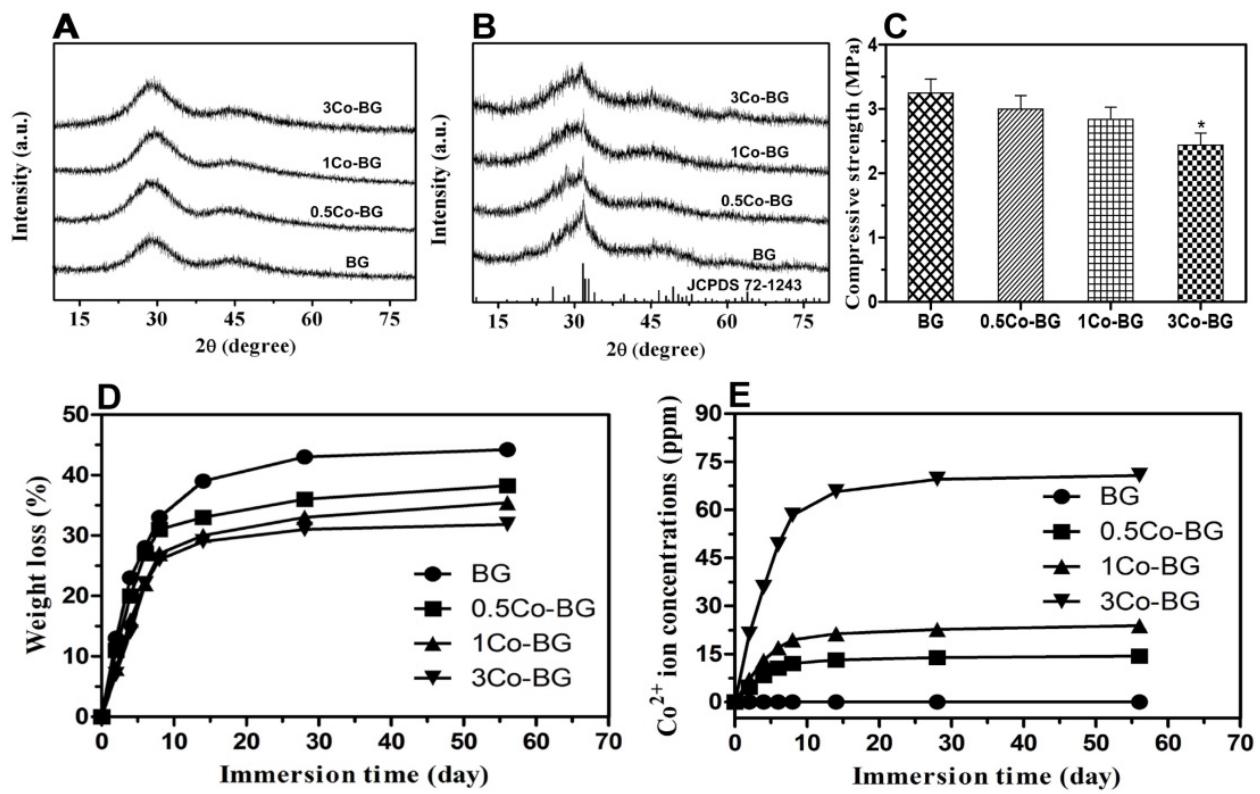

Figure 2. XRD diffraction patterns of BG, 0.5Co-BG, 1 Co-BG and 3Co-BG scaffolds before and after soaking in SBF for 90 days. For comparison, the patterns of the as-fabricated BG scaffold and a reference hydroxyapatite (JCPDS 72-1243) are also shown(A,B). The mechanical behavior of BG, 0.5Co-BG, 1Co-BG and 3Co-BG scaffolds $(C)$. Weight loss of the four groups of bioactive glass scaffolds shown and (D) the cumulative amount of $\mathrm{Co}^{2+}$ ions release from the glass into SBF as a function of immersion time of the scaffolds in $\mathrm{SBF}(\mathrm{E})$.

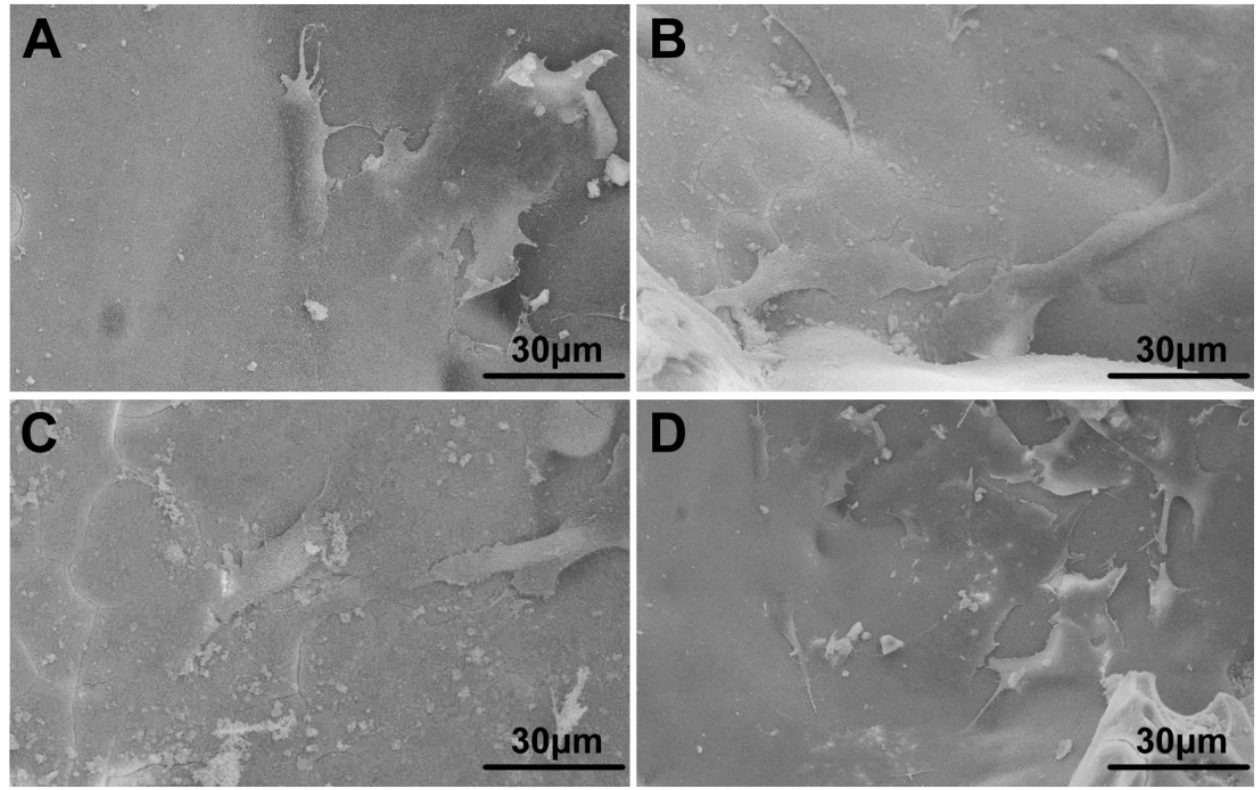

Figure 3. FESEM images of the attachment of hBMSCs on the BG (A), 0.5Co-BG (B), 1Co-BG (C) and 3Co-BG (D) scaffolds after incubation for 3 days. 
A

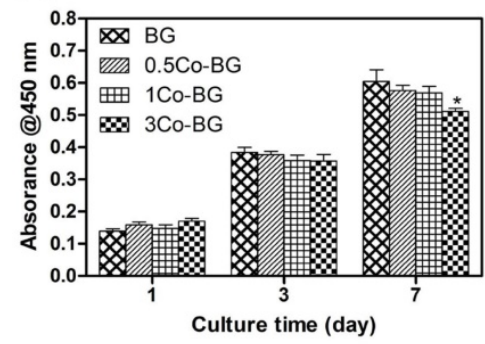

B

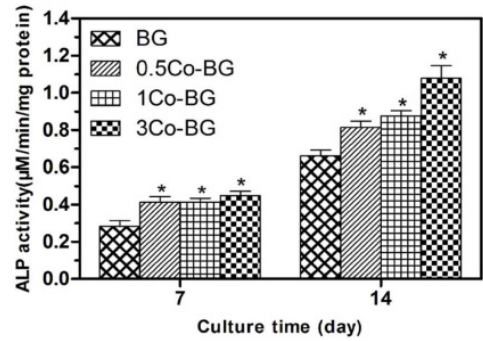

C
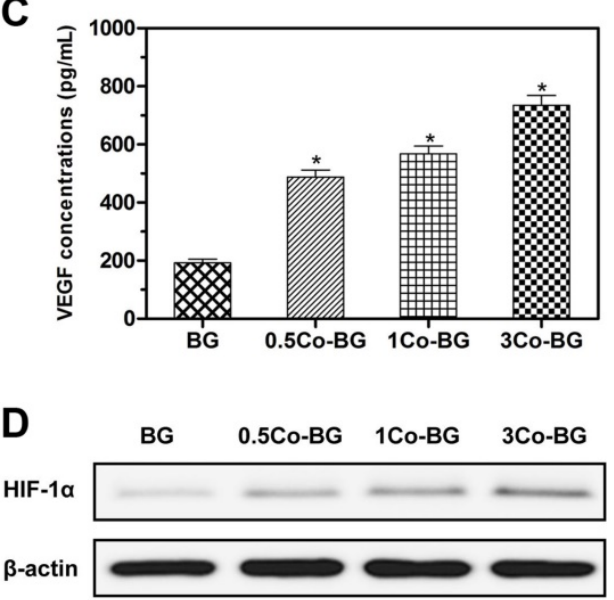

Figure 4. (A) Quantitative analysis of the proliferation of hBMSCs cultured on the BG, 0.5Co-BG, 1Co-BG and 3Co-BG scaffolds shown for 1,3 and 7 days. Mean $S D ; n=3$. *Significant difference when compared to BG ( $p<0.05$ ). (B) ALP activity of hBMSCs cultured for 7 and 14 days on the BG, 0.5 Co-BG, 1 co-BG and 3Co-BG scaffolds shown. Mean SD; $n=3$. *Significant difference when compared to BG $(p<0.05)$. (C) The effect of Co contents in the scaffolds on VEGF secretion of $h B M S C s$. Mean SD; $n=3$. *Significant difference when compared to BG $(p<0.05)$. (D) HIF-1 $\alpha$ expression of hBMSCs on the BG, 0.5Co-BG, 1Co-BG and 3Co-BG scaffolds by western blotting.

The angiogenesis/osteogenesis related genes, for example, bFGF, VEGF, SDF-1, BMP-2 and RUNX2, expression of the cultured hBMSCs were increased at both incubation periods (Fig. 5). All these angiogenesis/osteogenesis related genes were upregulated as the increase Co doping amount. These results showed that the addition of Co in BG can promote the osteogenic differentiation and angiogenesis of hBMSCs, and the 3Co-BG scaffolds had the strongest ability to help angiogenesis and osteogenic differentiation.

\section{Characterization of blood formation in vivo}

The reconstructed Micro-CT image (Fig. 6A) showed that the vascular density, number and area of the 3Co-BG scaffolds were much higher than that of BG or unfilled defects (Fig. 6, B and C) when the bioactive glass scaffolds or blank scaffolds were implanted in the calvarial defect of rats.

Immunofluorescence staining and immunohistochemistry for CD31 were used to detect the neovascularization in the defect area. And the blood vessels were oval structure or typically round and red or positive CD31 stain. The number of blood vessels at the implantation defect of the 3Co-BG scaffolds was greater than that at the $B G$ scaffolds or the unfilled defects (Fig. 7A, B, C, G, H and I), which was consistent with the above Micro-CT data.

\section{Evaluation of new bone tissues regeneration in vivo}

The Micro-CT results exhibited that the number of new tissues on the defects of the 3Co-BG scaffolds was greater than that on the unfilled defects or on the BG scaffolds (Fig. 8A, top and bottom view). Also, the gap between host bone and the edge of BG scaffolds was evident. Similarly, the new regeneration tissue amount of 3Co-BG scaffolds were higher than that of BG scaffolds (Fig. 8A, lateral view). Moreover, the new bone regeneration of 3Co-BG samples was significantly better than that of $\mathrm{BG}$ samples by BMD and BV/TV data (Fig. 8B, C).

The new bone mineralization and formation amount of scaffolds were demonstrated in Fig.9A, and the histological morphology of unfilled defects was determined by quantitative polychrome sequential fluorescent labeling tetracycline. Percent tetracycline labeling of 3 Co-BG $(2.7 \pm 0.5 \%)$ were markedly greater than BG group $(1.8 \pm 0.3 \%)$, in turn, further greater than untreated defects $(0.6 \pm 0.2 \%)$ at 2 week. In the 4 week, the alizarin red label (red, Fig. 9, column 2) 
shown at BG, 3Co-BG and unfilled defects was $2.6 \pm$ $0.6 \%, 4.9 \pm 0.8 \%$ and $1.0 \pm 0.2 \%$ severally. The difference between two comparisons among these samples was statistically significant $(p<0.05)$, and the results went similar trend at week 6 and 8 .

Van Gieson's staining analysis indicated similar bone regeneration trend which was in line with the previous data of fluorescence labeling and Micro-CT. New bone formation was abundant around the 3Co-BG scaffolds, but relatively low on the BG scaffolds, with the least new bone formation in the unfilled defects (Fig. 10A). The new bone area was 48 $\pm 5 \%, 24 \pm 6 \%$ and $4 \pm 3 \%$ orderly in 3 Co-BG, BG and unfilled defects (Fig. 10B). The differences among the groups were statistically significant $(\mathrm{p}<0.05)$.

Immunofluorescence analysis at the unfilled defects revealed nearly no staining of OCN (Fig. 7 $\mathrm{D}, \mathrm{E}, \mathrm{F}, \mathrm{J}, \mathrm{K}$ and $\mathrm{L}$ ) at week 8 . In contrast, some of the OCN positive staining was discovered on BG scaffolds, while a large number of OCN positive staining was found around the 3Co-BG scaffolds. These results indicated that 3 Co-BG scaffolds were
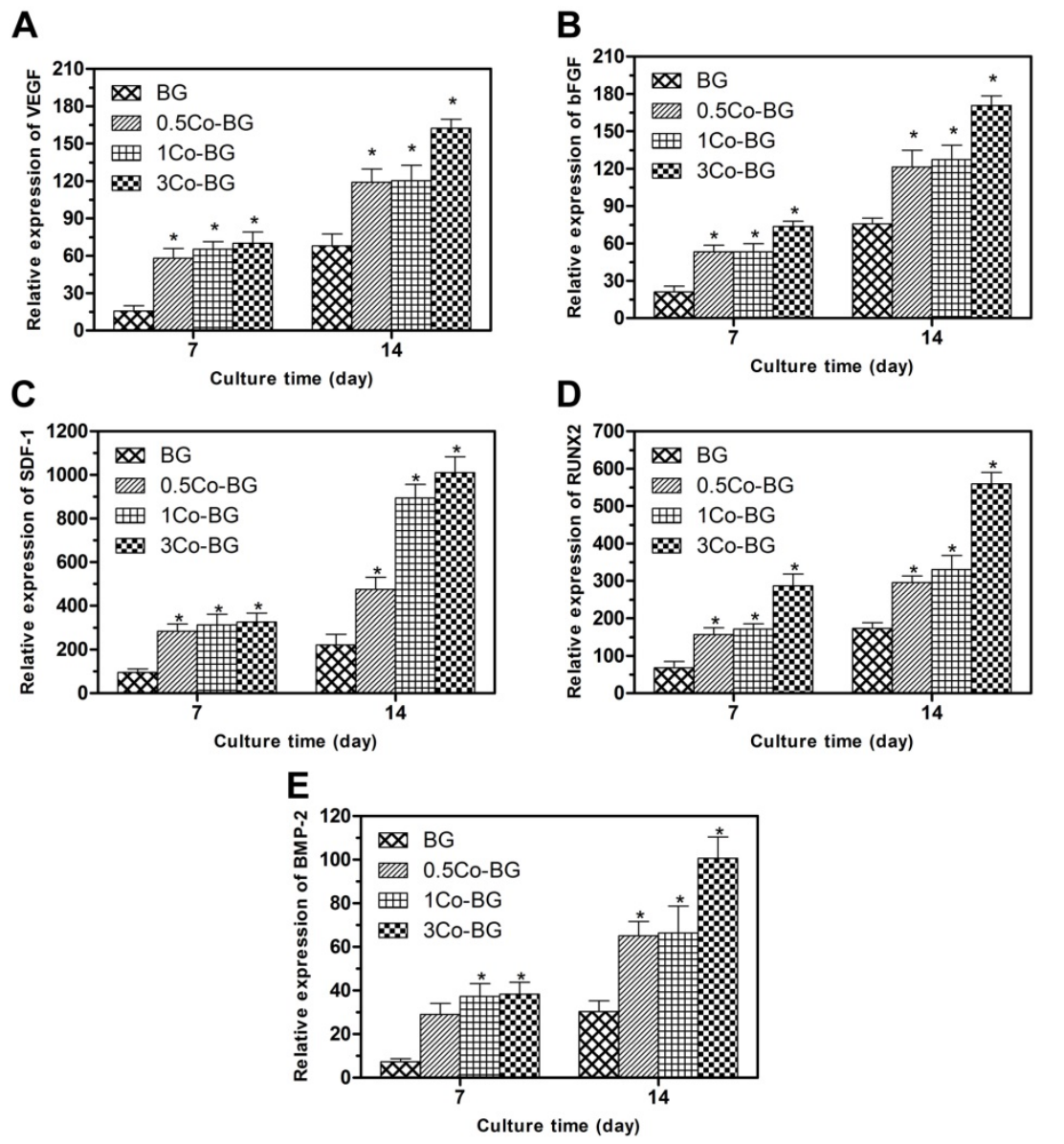

Figure 5. Gene expression, as measured by qRT-PCR, of the angiogenic genes VEGF (A), bFGF (B) and SDF-1 (C), and the osteogenic genes RUNX2 (D) and BMP-2 (E) for $\mathrm{hBMSCs}$ cultured for 7 and 14 days on the BG, 0.5Co-BG, 1Co-BG and 3Co-BG scaffolds shown. Mean SD; $\mathrm{n}=3$. *Significant difference when compared to BG $(p<0.05)$. 
A
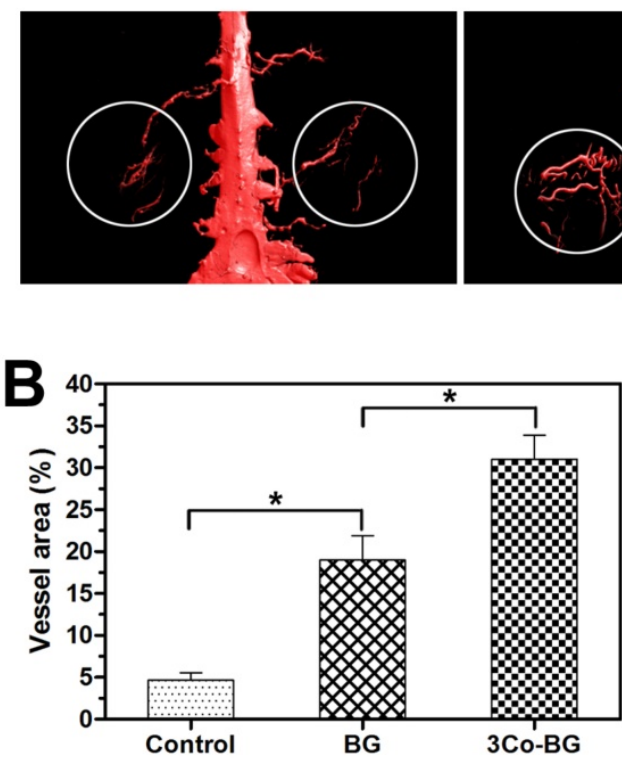

BG
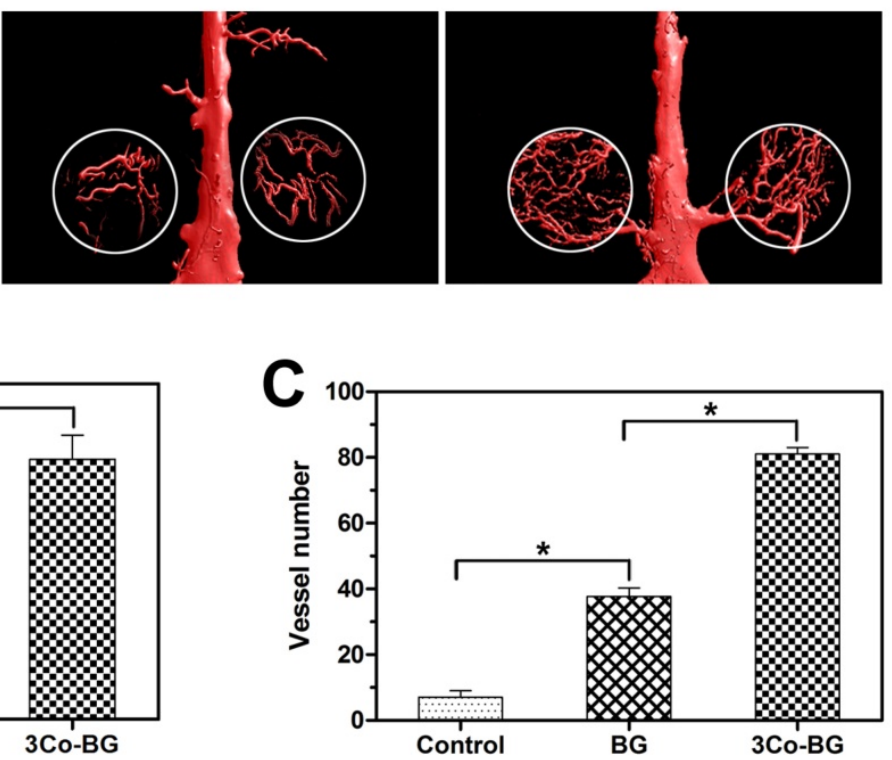

Figure 6. Micro-CT evaluation of blood vessel formation in rat calvarial defects implanted for 8 weeks with the BG and 3 Co-BG scaffolds and in the unfilled defects (control). (A) 3D reconstructed images of the new blood vessels; (B), (C) Morphometric analysis of the new blood vessel area and the number of blood vessels. Mean $\pm \mathrm{SD} ; \mathrm{n}=3$. *Significant difference between groups $(\mathrm{p}<0.05)$.

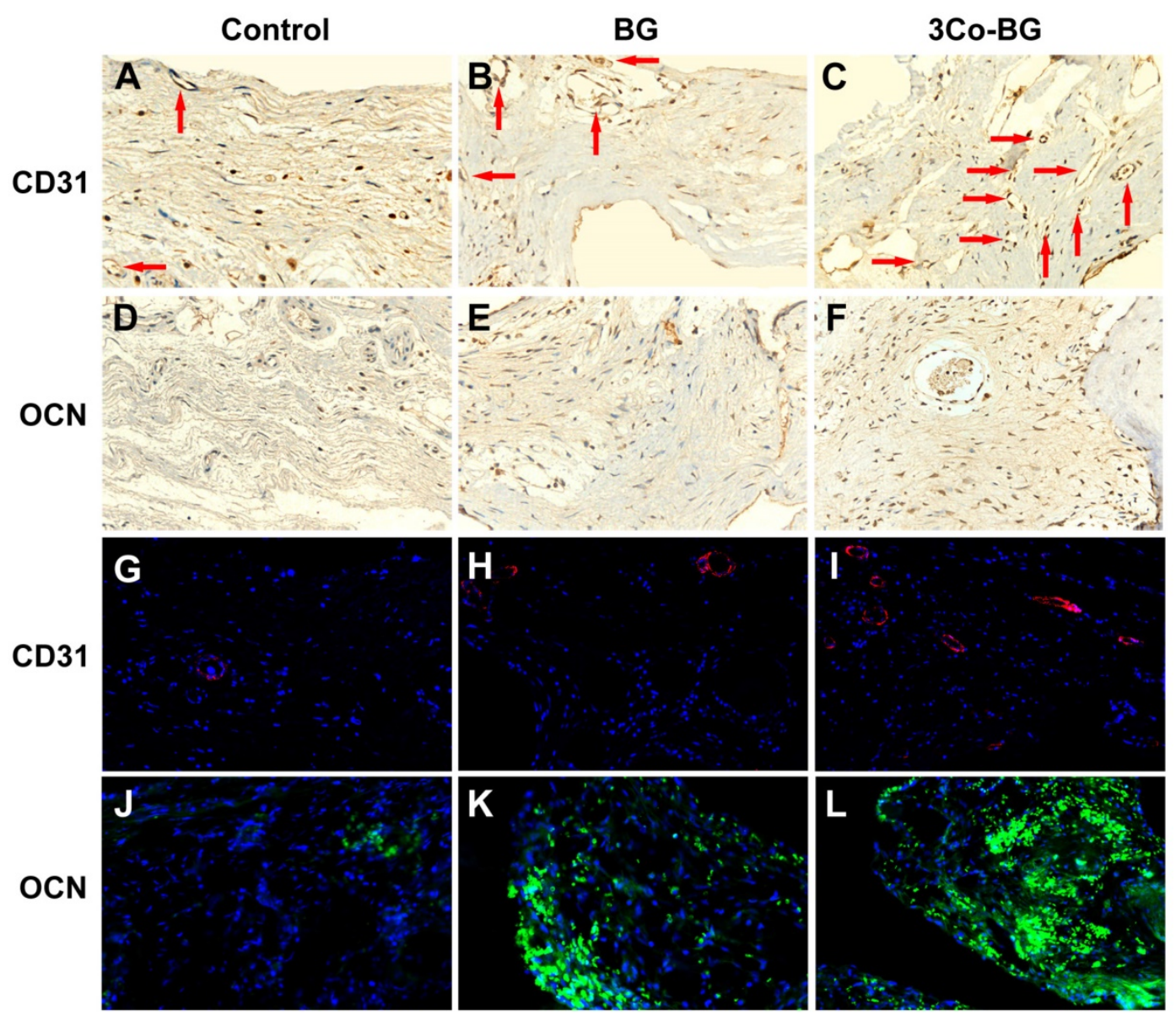

Figure 7. Immunohistochemical and immunofluorescence staining for CD31 and OCN in the defects implanted with the BG and 3 Co-BG scaffolds and in the unfilled defects (control) at 8 weeks post-implantation. Blood vessels were defined by positive CD31 staining or red fluorescence and their typical round or oval structure. There were more new vessels (red arrows and red fluorescence) in the defects implanted with the 3 Co-BG scaffolds (C, I) than for the BG group (B, $H$ ) or the control group (A, G). There were almost no positive staining or green fluorescence for OCN in the control group (D, J), a limited amount in the BG group (E, K), and a higher amount 3Co-BG group (F, L). 
A

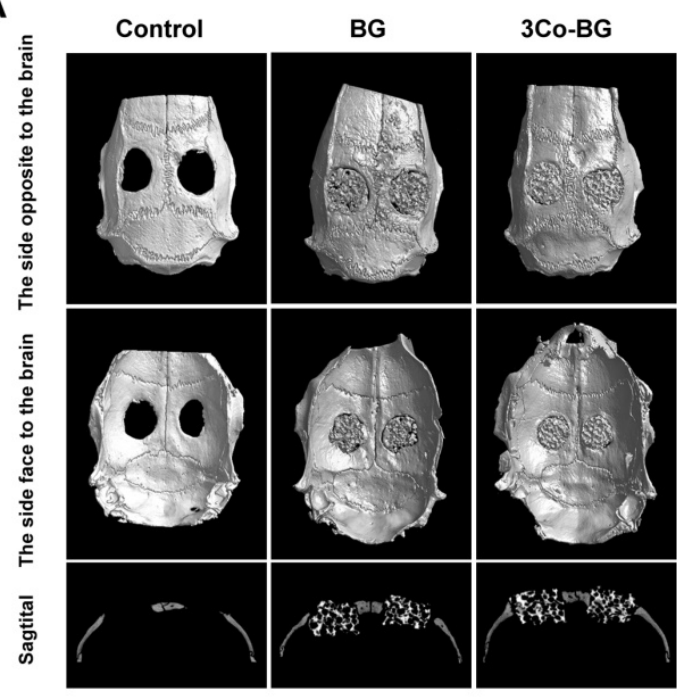

B

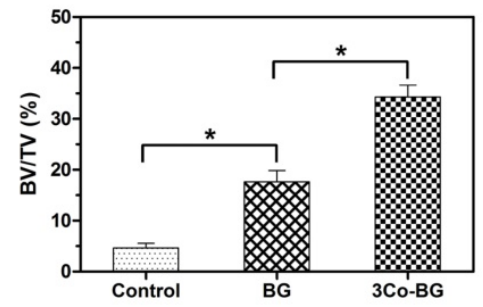

C

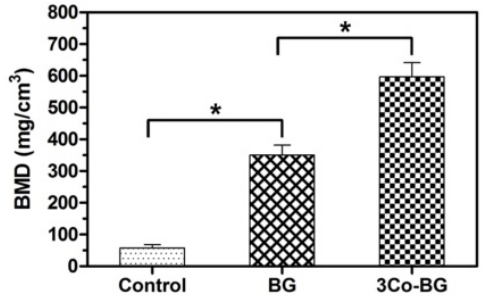

Figure 8. Micro-CT evaluation of bone regeneration in the rat calvarial defects implanted with the BG and the 3 Co-BG scaffolds and in the unfilled defect (control) at 8 weeks post-implantation. (A) Top, bottom and cross-sectional views of reconstructed images; (B, C) Bone mineral density (BMD) and bone volume/total volume $(B V / T V)$ in the defects implanted with the scaffolds and in the unfilled defects. Mean SD; $n=3$. *Significant difference between groups ( $<<0.05)$.
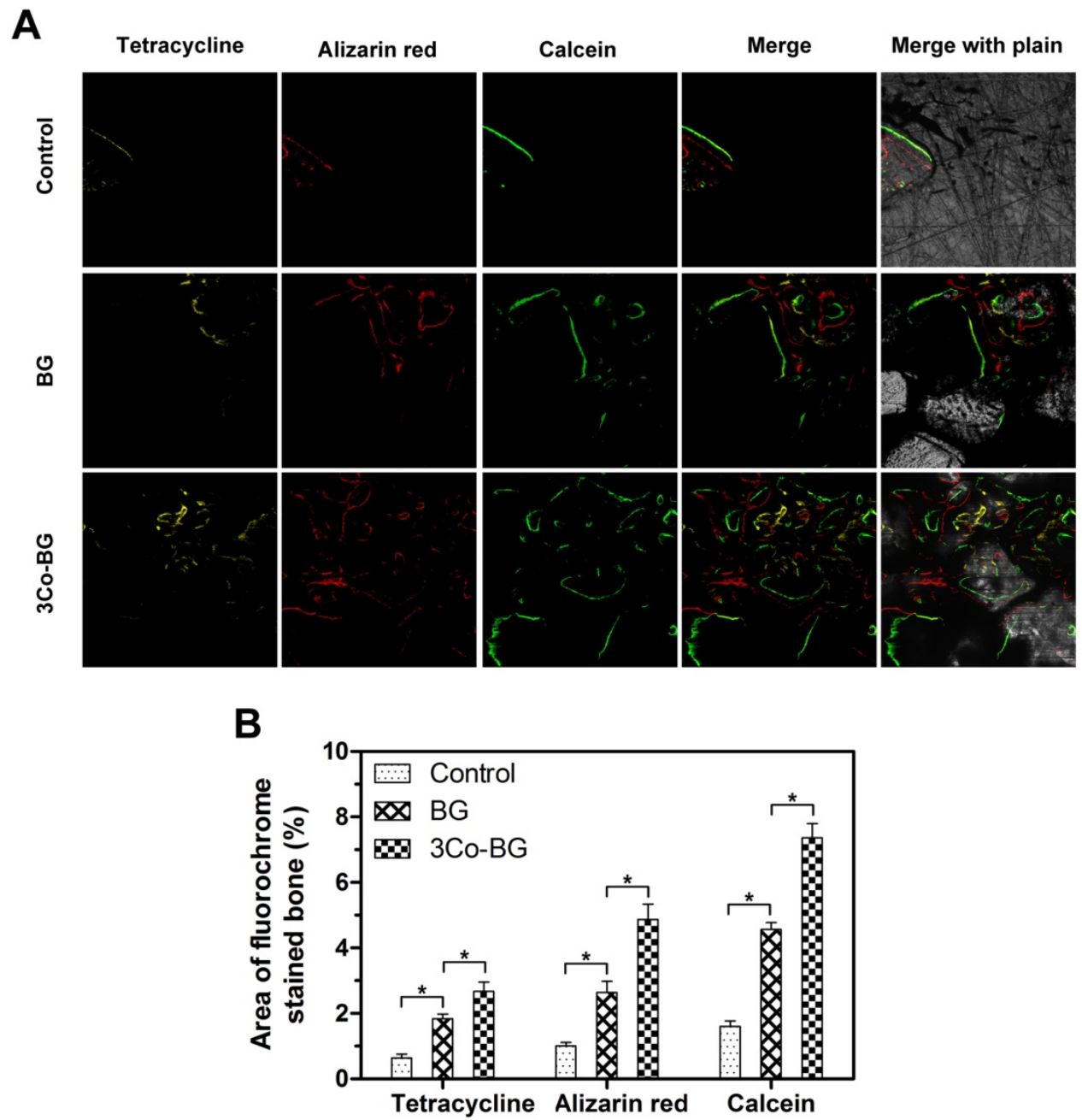

Figure 9. New bone formation and mineralization, determined histomorphometrically by fluorochrome-labeling analysis, in rat calvarial defects implanted with the BG and the 3Co-BG scaffolds and in the unfilled defects (control). (A) Column 1 (yellow) shows tetracycline at week 2; column 2 (red) shows alizarin red at week 4; column 3 (green) shows calcein at week 6; column 4 represents merged images of the three fluorochromes for the same group, and column 5 represents merged images from a plain microscope. (B) The percent fluorochrome area in the defects implanted with the scaffolds and the unfilled defects. Mean SD; $\mathrm{n}=3$. *Significant difference between groups $(p<0.05)$. 


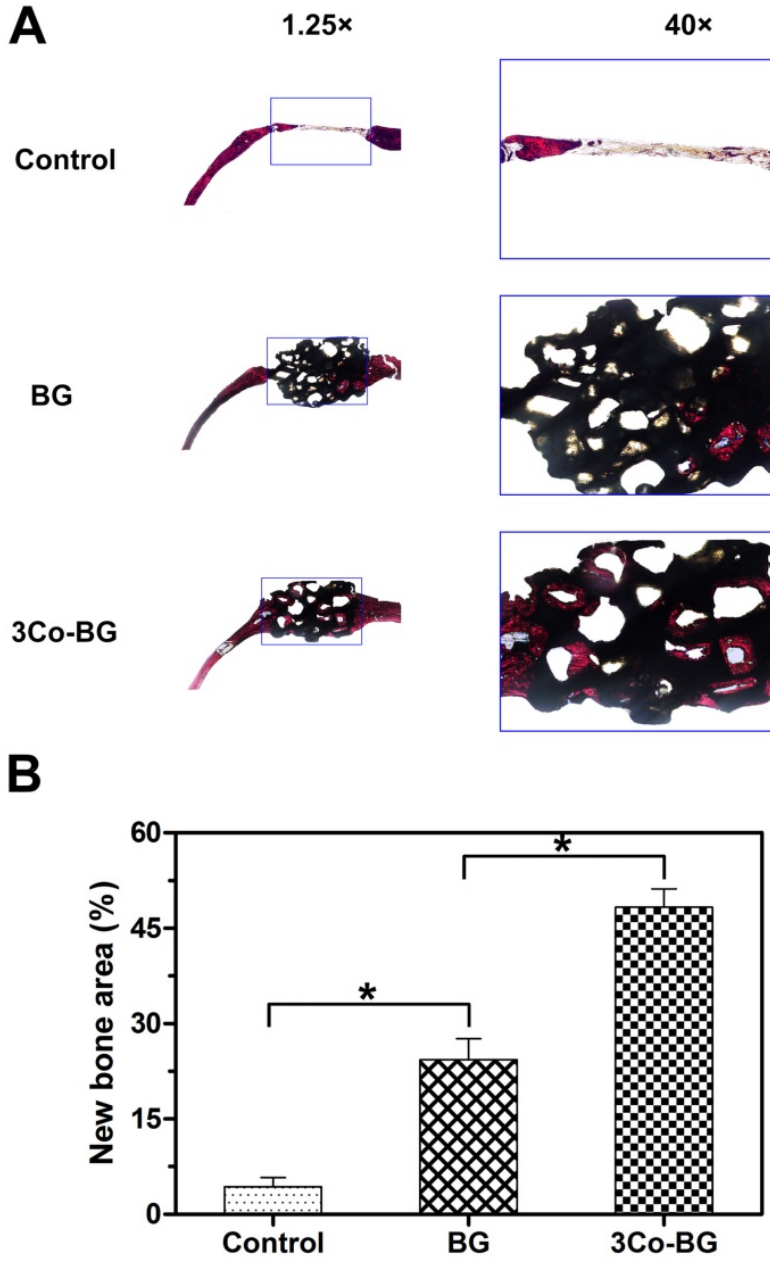

Figure 10. (A) Transmitted light images of $V a n$ Gieson picrofuchsin-stained sections of rat calvarial defects implanted with BG and 3 Co-BG scaffolds and the unfilled defects (control) at 8 weeks post-implantation. New bone appears red whereas the scaffold appears black. (B) Percent new bone area in the defects implanted with the scaffolds and in the unfilled defects. Mean SD; $n=3$. *Significant difference between groups $(p<0.05)$.

Additionally, the Co-doped BG scaffolds had a porosity of $80 \pm 3 \%$ used in the present study, interconnected pores of size 200-500 $\mu \mathrm{m}$. The microstructural are benifit for oxygen and nutrient transport, attachment, migration and proliferation of bone-forming cells, vascularization and bone in growth. ${ }^{[32]}$ The scaffolds showed a sustained and controllable release ability in vitro. Rather, a concern is that high concentrations of Co can cause toxicity to cells in vitro and pathologies in vivo.[33][34] [35]Thus, this controlled release materials is very suitable for vascularized bone regeneration in tissue engineering applications.

For the cell responds results, Co-BG scaffolds can well support the proliferation of hBMSCs (Fig. 3). Quantitation of hBMSCs proliferation using the CCK-8 assay showed no dramatically significant differences among groups at the incubation times used (1, 3 and 7 days) with the hBMSCs proliferation of 3Co-BG scaffolds which was inconspicuously lower only at day 7.

The Co-doped bioactive glass scaffolds revealed the ability to significantly enhance the ALP activity and osteogenic gene (RUNX2 and BMP-2) expression of the hBMSCs during the fourteen-day incubation period (Fig. 5 and 6). Additionally, the Co-doped scaffolds induced a significant hypoxic cascade, including increased VEGF protein secretion, HIF-1a and angiogenic gene (VEGF, bFGF and SDF-1) expression of the hBMSCs cultured on the scaffolds. VEGF, a identified key regulator for angiogensis ${ }^{[12][36][37]}$ can affect endothelial cells by stimulating their liberation, migration, proliferation and, eventually, the formation of tubular structures. ${ }^{[38]}$ HIF-1a can initiate the expression of kinds of angiogenic genes associated with bone tissue development and regeneration. ${ }^{[13][14][39]}$ Although oxygen is present in normoxic conditions, $\mathrm{Co}^{2+}$ ions can deactivate HIF-specific PHD, leading to the stabilization of HIF-1a and, thus, to mimicking the hypoxia condition. ${ }^{[40]}$

The 3Co-BG scaffolds exhibited the capacity to significantly enhance the regeneration of new bone tissue and the formation of new blood vessel formation compared BG scaffolds. As it reported that hypoxia plays an key role in improving angiogenesis and osteogenesis by activating the HIF-1a pathway and manipulating the behavior of MSCs, including their migration, proliferation, differentiation and growth factor secretion. ${ }^{10]}$ [12] [40] Rosova'et al. suggested that pre-culturing MSCs in hypoxic conditions increased their potential to migrate and to regenerate tissue. ${ }^{[41]}$ Okuyama et al. found that VEGFR1 expression was upregulated by HIF-1a after culturing MSCs in hypoxic conditions. ${ }^{[42]}$ Also, MSCs have been reported to facilitate implant vascularization by producing a variety of growth factors under hypoxic conditions.[43] Previous studies also showed that pharmacologic stimulation of HIF-1a activity by the application of $\mathrm{Co}^{2+}$ ions was capable of inducing a hypoxic cascade of MSCs and induced a robust angiogenic response that was coupled to a subsequent osteogenic response. [10] [20] [39]

In the present study, the hypoxic-like condition created by released $\mathrm{Co}^{2+}$ in vivo hypothetically motivated HIF-1a proteins to bind to HIF-1 $\beta$. The very binding could further initiate the transcription of some hypoxia-sensitive genes, then in treturn, these genes receptors could induce the higher expression of osteogenesis and angiogenesis relative genes. ${ }^{[44]}[45]$ Briefly, with the increased concentration of $\mathrm{Co}^{2+}$ ions, the more expression of HIF-1a (Fig. 4), ALP (Fig. 5), VEGF, bFGF, SDF-1, RUNX2 and BMP-2 (Fig. 6) were characterized from protein and gene perspective. Meanwhile, the new well-reconstructed blood vessels (Fig. 7) and the new bone regeneration, formation and 
mineralization (Fig. 9, 10 and 11). In the end, the high expression osteogenesis genes could further promote the secretion of VEGF, which in turn stimulates angiogenesis.[46][47] So, these highly co-expressed osteogenesis and angiogenesis relative factors promoted cell ossification. ${ }^{[48]}\left[{ }^{49]}\right.$ In conclusion, $\mathrm{Co} 2+$ ion, a inhibitor of PHD, promotes the regeneration of blood vessels and bone via activating HIF-1a and related genes.

\section{Conclusions}

Porous scaffolds of BG scaffolds doped with 0.5-3.0 wt\% CoO released $\mathrm{Co}^{2+}$ ions and converted to hydroxyapatite when soaked in SBF in vitro. The cumulative amount of $\mathrm{Co}^{2+}$ ions released was dependent on the amount of $\mathrm{CoO}$ in the as-prepared glasses, which indicates the ability to control the $\mathrm{Co}^{2+}$ ion release at therapeutically acceptable levels. At the concentrations used, the Co in the scaffolds was not cytotoxic and it notably promoted VEGF protein secretion, HIF-1a expression, ALP expression of osteogenic and angiogenic genes of hBMSCs in vitro. The 3Co-BG scaffolds significantly enhanced blood vessel formation and bone regeneration in rat calvarial defects at 8 weeks post-implantation. Together, the results show that the biomaterial implantation of controlled and sustained release of $\mathrm{Co}^{2+}$ ions is a promising strategy to stimulate neovascularization in the repairing of osseous defects.

\section{Acknowledgments}

The authors acknowledge the support by the Innovation Found of Precision Medicine (Grant No.IF PM2016A003), and National Natural Science Foundation of China (No.51672191, 51802326, 81871774), and funds from Health Bureau of Zhejiang Province (No. 2018KY192).

\section{Competing Interests}

The authors have declared that no competing interest exists.

\section{References}

1. Bauer TW, Muschler GF. Bone Graft Materials: An Overview of the Basic Science. Clinical Orthopaedics \& Related Research. 2000; 371: 10.

2. Arcos D, Izquierdo-Barba I, Vallet-Regi M. Promising trends of bioceramics in the biomaterials field. J Mater Sci Mater Med. 2009; 20: 447-55.

3. Zhang J, Zhao S, Zhu Y, Huang Y, Zhu M, Tao C, et al. Three-dimensional printing of strontium-containing mesoporous bioactive glass scaffolds for bone regeneration. Acta Biomaterialia. 2014; 10: 2269-81.

4. Pei P, Qi X, Du XY, Zhu M, Zhao SC, Zhu YF. Three-dimensional printing of tricalcium silicate/mesoporous bioactive glass cement scaffolds for bone regeneration. J Mater Chem B. 2016; 4: 7452-63.

5. Hao D, You-Shui G, Yang W, Chen H, Yuan S, Changqing Z. Dimethyloxaloylglycine increases the bone healing capacity of adipose-derived stem cells by promoting osteogenic differentiation and angiogenic potential. Stem Cells \& Development. 2014; 23: 990-1000.

6. Azevedo MM, Jell G, O'Donnell MD, Law RV, Stevens MM. Synthesis and Characterization of Hypoxia-Mimicking Bioactive Glasses for Skeletal Regeneration. J Mater Chem. 2010; 20: 8854.
7. Peng P, Wei D, Min Z, Du X, Zhu Y. The effect of calcium sulfate incorporation on physiochemical and biological properties of 3D-printed mesoporous calcium silicate cement scaffolds. Microporous \& Mesoporous Materials. 2017; 241: 11-20.

8. Yu H, Vandevord PJ, Mao L, Matthew HW, Wooley PH, Yang SY. Improved tissue-engineered bone regeneration by endothelial cell mediated vascularization. Biomaterials. 2009; 30: 508-17.

9. Portal-Nunez S, Lozano D, Esbrit P. Role of angiogenesis on bone formation. Histol Histopathol. 2012; 27: 559-66.

10. Fan W, Crawford R, Xiao Y. Enhancing in vivo vascularized bone formation by cobalt chloride-treated bone marrow stromal cells in a tissue engineered periosteum model. Biomaterials. 2010; 31: 3580-9.

11. Giuseppina B, Francesca G, Simona B, Laura B, Federica B, Marilena M, et al. Hypoxia stimulates the production of the angiogenesis inhibitor 2-methoxyestradiol by swine granulosa cells. Steroids. 2011; 76: 1433-6.

12. Sunday O, Song H, Joanna K, Junjie Y, Andrea S, Sohn RE, et al. VEGF is essential for hypoxia-inducible factor-mediated neovascularization but dispensable for endothelial sprouting. P Natl Acad Sci USA. 2011; 108: 13264-9.

13. Pugh CW, Ratcliffe PJ. Regulation of angiogenesis by hypoxia: role of the HIF system. Nat Med. 2003; 9: 677-84.

14. Zou D, Zhang Z, He J, Zhang K, Ye D, Han W, et al. Blood vessel formation in the tissue-engineered bone with the constitutively active form of HIF-1a mediated BMSCs. Biomaterials. 2012; 33: 2097-108.

15. Fong GH. Mechanisms of adaptive angiogenesis to tissue hypoxia. Angiogenesis. 2008; 11: 121-40.

16. Min JH, Yang H, Ivan M, Gertler F, Kaelin WG, Pavletich NP. Structure of an HIF-1a-pVHL Complex: Hydroxyproline Recognition in Signaling. Science. 2002; 296: 1886-9.

17. Cheng L, An-Ly T, Yen-Chu C, Shih-Chen F, Chun-Hsien H, Chia-Ching W, et al. Facilitation of human osteoblast apoptosis by sulindac and indomethacin under hypoxic injury. J Cell Biochem. 2011; 113: 148-55.

18. Zhu M, Zhao SC, Xin C, Zhu YF, Zhang CQ. 3D-printed dimethyloxallyl glycine delivery scaffolds to improve angiogenesis and osteogenesis. Biomater Sci-Uk. 2015; 3: 1236-44.

19. Xing S, Chao W, Girish R, Mahendra M, Ying W, Duvall CL, et al. Prolyl hydroxylase inhibitors increase neoangiogenesis and callus formation following femur fracture in mice. J Orthop Res. 2010; 27: 1298-305.

20. Chao W, Gilbert SR, Ying W, Xuemei C, Xing S, Girish R, et al. Activation of the hypoxia-inducible factor-1alpha pathway accelerates bone regeneration. Proc Natl Acad Sci U S A. 2008; 105: 686-91.

21. Hoppe A, Jokic B, Janackovic D, Fey T, Greil P, Romeis S, et al. Cobalt-releasing 1393 bioactive glass-derived scaffolds for bone tissue engineering applications. Acs Appl Mater Interfaces. 2014; 6: 2865-77.

22. Wu C, Zhou Y, Fan W, Han P, Chang J, Yuen J, et al. Hypoxia-mimicking mesoporous bioactive glass scaffolds with controllable cobalt ion release for bone tissue engineering. Biomaterials. 2012; 33: 2076-85.

23. Huang W, Day DE, Kittiratanapiboon K, Rahaman MN. Kinetics and mechanisms of the conversion of silicate (45S5), borate, and borosilicate glasses to hydroxyapatite in dilute phosphate solutions. Journal of Materials Science Materials in Medicine. 2006; 17: 583-96.

24. Rahaman MN, Day DE, Bal BS, Fu Q, Jung SB, Bonewald LF, et al. Bioactive glass in tissue engineering. Acta Biomaterialia. 2011; 7: 2355-73.

25. Fu Q, Rahaman MN, Fu H, Liu X. Silicate, borosilicate, and borate bioactive glass scaffolds with controllable degradation rate for bone tissue engineering applications. I. Preparation and in vitro degradation. Journal of Biomedical Materials Research Part A. 2010; 95A: 172-9.

26. Masoud S, Samad N. A protocol for isolation and culture of mesenchymal stem cells from mouse bone marrow. Nat Protoc. 2009; 4: 102-6.

27. Zhu Z, Yin J, Guan J, Hu B, Niu X, Jin D, et al. Lithium stimulates human bone marrow derived mesenchymal stem cell proliferation through GSK-3 $\beta$-dependent $\beta$-catenin/Wnt pathway activation. Febs Journal. 2015; 281: 5371-89.

28. Kawashita M, Shineha R, Kim HM, Kokubo T, Inoue Y, Araki N, et al. Preparation of ceramic microspheres for in situ radiotherapy of deep-seated cancer. Biomaterials. 2003; 24: 2955-63.

29. Kelly BD, Hackett SF, Kiichi H, Yuji O, Zheqing C, Shannon BD, et al. Cell type-specific regulation of angiogenic growth factor gene expression and induction of angiogenesis in nonischemic tissue by a constitutively active form of hypoxia-inducible factor 1. Circ Res. 2003; 93: 1074-81.

30. Kannan RY, Salacinski HJ, Sales K, Butler P, Seifalian AM. The roles of tissue engineering and vascularisation in the development of micro-vascular networks: a review. Biomaterials. 2005; 26: 1857-75.

31. Sun X, Kang Y, Bao J, Zhang Y, Yang Y, Zhou X. Modeling vascularized bone regeneration within a porous biodegradable $\mathrm{CaP}$ scaffold loaded with growth factors. Biomaterials. 2013; 34: 4971-81.

32. Karageorgiou V, Kaplan D. Porosity of 3D biomaterial scaffolds and osteogenesis. Biomaterials. 2005; 26: 5474-91.

33. Cyrille F, Alain P, Fackson M, John A, Zukor DJ, Maryam T, et al. Effect of cobalt and chromium ions on human MG-63 osteoblasts in vitro: morphology, cytotoxicity, and oxidative stress. Biomaterials. 2006; 27: 3351-60.

34. Daou S, Chemaly AE, Christofilopoulos P, Bernard L, Hoffmeyer P, Demaurex $\mathrm{N}$. The potential role of cobalt ions released from metal prosthesis on the inhibition of Hv1 proton channels and the decrease in Staphyloccocus epidermidis killing by human neutrophils. Biomaterials. 2011; 32: 1769-77. 
35. Shrivastavaa K, Singh B, Sairam M, Ilavazhagan G. Sub-chronic oral toxicity study in Sprague-Dawley rats with hypoxia mimetic cobalt chloride towards the development of promising neutraceutical for oxygen deprivation. Experimental \& Toxicologic Pathology. 2010; 62: 489-96.

36. Lode A, Reinstorf WB. Calcium phosphate bone cements, functionalized with VEGF: release kinetics and biological activity. Journal of Biomedical Materials Research Part A. 2010; 81A: 474-83.

37. Ferrara N, Davissmyth $\mathrm{T}$. The biology of vascular endothelial growth factor; 1996.

38. Soker S, Machado M, Atala A. Systems for therapeutic angiogenesis in tissue engineering. World J Urol. 2000; 18: 10-8.

39. Xinyang H, Ping YS, Fraser JL, Zhongyang L, Ogle ME, Jian-An W, et al. Transplantation of hypoxia-preconditioned mesenchymal stem cells improves infarcted heart function via enhanced survival of implanted cells and angiogenesis. Journal of Thoracic \& Cardiovascular Surgery. 2008; 135: 799-808.

40. Hewitson KS, Mcneill LA, Riordan MV, Ya-Min T, Bullock AN, Welford RW, et al. Hypoxia-inducible factor (HIF) asparagine hydroxylase is identical to factor inhibiting HIF (FIH) and is related to the cupin structural family. J Biol Chem. 2002; 277: 26351-5.

41. Ivana R, Mo D, Ben C, Daniel L, Nolta JA. Hypoxic preconditioning results in increased motility and improved therapeutic potential of human mesenchymal stem cells. Stem Cells. 2010; 26: 2173-82.

42. Hiroaki O, Balaji K, Fu ZY, Hideko N, Marta BM, Semenza GL. Expression of vascular endothelial growth factor receptor 1 in bone marrow-derived mesenchymal cells is dependent on hypoxia-inducible factor 1 . J Biol Chem. 2006; 281: 15554-63.

43. SC H, RR P, SC C, SC H, DJ P. Angiogenic effects of human multipotent stromal cell conditioned medium activate the PI3K-Akt pathway in hypoxic endothelial cells to inhibit apoptosis, increase survival, and stimulate angiogenesis. Stem cells (Dayton, Ohio). 2007; 25: 2363-70.

44. Yanqiu L, Berendsen AD, Shidong J, Sutada L, Roland B, Napoleone F, et al. Intracellular VEGF regulates the balance between osteoblast and adipocyte differentiation. J Clin Invest. 2012; 122: 3101-13.

45. Li XD, Chen J, Ruan CC, Zhu DL, Gao PJ. Vascular endothelial growth factor-induced osteopontin expression mediates vascular inflammation and neointima formation via Flt-1 in adventitial fibroblasts. Arteriosclerosis Thrombosis \& Vascular Biology. 2012; 32: 2250.

46. Deckers MML, Bezooijen RL, Van, Geertje VDH, Jakomijn H, Chris VDB, Papapoulos SE, et al. Bone morphogenetic proteins stimulate angiogenesis through osteoblast-derived vascular endothelial growth factor A. Endocrinology. 2002; 143: 1545-53.

47. G C, S J, GC K. Osteopontin promotes vascular endothelial growth factor-dependent breast tumor growth and angiogenesis via autocrine and paracrine mechanisms. Cancer Res. 2008; 68: 152-61.

48. Keramaris N, Calori G, Nikolaou V, Eh, Giannoudis P. Fracture vascularity and bone healing: a systematic review of the role of VEGF. Injury. 2008; 39: S45-S57.

49. Masanori N, Akiko N, Max VL, Hiroyuki Y, Sarah H, Drexler HCA, et al. Spatial regulation of VEGF receptor endocytosis in angiogenesis. Nat Cell Biol. 2013; 15: 249-60. 